\title{
Economic Analysis of Networking Technologies for Rural Developing Regions
}

\author{
Shridhar Mubaraq Mishra*, John Hwang ${ }^{+}$, Dick Filippini ${ }^{+}$, Reza Moazzami ${ }^{+}$, \\ Lakshminarayanan Subramanian* and Tom $\mathrm{Du}^{+}$ \\ * EECS Department ${ }^{+}$Haas School of Business \\ UC Berkeley UC Berkeley
}

\begin{abstract}
Providing network connectivity to rural regions in the developing world is an economically challenging problem especially given the low income levels and low population densities in such regions. Many existing connectivity technologies incur a high deployment cost that limits their affordability. Leveraging several emerging wireless technologies, this paper presents the case for economically viable networks in rural developing regions. We use the Akshaya Network located in Kerala, India as a specific case study. and show that a wireless network using WiFi for the backhaul, CDMA450 for the access network, and shared PCs for end user devices has the lowest deployment cost. However, if we include the expected spectrum licensing cost for CDMA450, a network with lease exempt spectrum using WiFi for the backhaul and WiMax for access is the most economically attractive option. Even with license exemption, regulatory costs comprise nearly half the total cost in the WiFi/WiMax case suggesting the possibility of significant improvement in network economics with more favorable regulatory policies. Finally, we also demonstrate the business case for a WiFi/CDMA450 network with nearly fully subsidized cellular handsets as end user devices.
\end{abstract}

\section{Introduction}

The lack of network connectivity in many regions around the world is as much an economic problem as a technological one. This is strikingly true for rural regions in developing countries with low income levels and low population densities. On one hand, the presence of network connectivity within these regions can have potentially several far-reaching implications including promoting literacy, improving health care [1], reducing market inefficiencies [2], increasing government transparency [3] and enabling environmental monitoring [4]. On the other hand, traditional connectivity solutions using fiber-optic networks may be ill-suited for such environments. The high infrastructure cost and the long period of time to deploy fiber-optic networks make these technologies economically less appealing and a very risky investment proposition. Additionally, high bandwidth links may not be necessary in such regions since many of the applications (e.g. messaging, voice) leveraging the network connectivity have limited bandwidth requirements. For rural markets with dispersed populations and uncertain demand, network technologies with a low cost of entry are preferable. 
Long-distance wireless technologies, especially those based on standards can enable networking in rural regions. The attractive features of these networks include the low-deployment cost, ease of deployment and the ability to cater to a wide-range of geographic terrain. On the flip side, these wireless networks have capacity constraints that limit the maximum available bandwidth and also suffer from reliability problems. The capacity that a fiber-optic network provides is orders of magnitude larger than that of a backhaul wireless network. Hence, from an economic standpoint, the cost per bit of capacity for a fiber-optic network is much smaller than that of a wireless-backhaul network.

In this paper, we study the economic viability of different technologies for providing network connectivity in emerging regions. Specifically, we ask the question: What is the networking technology that can provide connectivity at the lowest cost per unit of usage while remaining profitable? This cost per unit of demand is distinct from the traditional focus on cost per unit of supply. The cost per unit of demand can be expressed as the ratio of the cost per user and the demand per user. Sharing of devices helps reduce cost per user significantly. However sharing devices does carry a penalty: lower expected demand per user.

Any such technology requires two connectivity components: the access network and the backhaul network. The access network provides connectivity within a local region and the backhaul network provides connectivity across regions. In this paper, we consider four forms of access technologies which exhibit different coverage and capacity characteristics: WiFi [5], WiMax [6], CDMA450 [7] and WipLL [8]. We consider these access technologies in combination with three forms of backhaul technologies: Fiber(PON) [9], WiFi [5] (with directional antennas) and VIP [10]. WipLL and VIP are proprietary technologies and will be discussed later in Section 3.

Our economic study is motivated by the Akshaya project in India whose aim is to provide Internet connectivity within a specific district in the state of Kerala, India. Based on economic data gathered from Akshaya, we analyze and compare the cost of various communication technologies for providing connectivity. For the Akshaya case, we show six key results. First, WiFi with directional antennas as backhaul technology combined with WiMax as an access technology provides the most attractive economics. However, if we discount the cost of spectrum licensing, WiFi/CDMA450 has a lower cost of deployment than WiFi/WiMax. Second, we found that the largest component of the capital investment for providing connectivity is the cost of the end-user devices (either PCs or cellular handsets). Third, tower and primary and/or backup power source costs dominate the last mile and backhaul costs. These costs are not expected to decrease in the future and hence any technology that is able to provide a large coverage area per tower or lower power consumption can reduce costs. Fourth, since network equipment comprises a small portion of the overall deployment cost, reducing equipment cost per node does not lead to significant improvement in economics. In fact, a counterintuitive conclusion of this analysis suggests that increasing the network equipment cost is desirable if it leads to greater coverage area/link distance and/or lower power consumption. Our analysis shows that if the coverage area of last mile devices could be doubled with an $4 \mathrm{x}$ increase in price, it would yield the same rate of return. Fifth, a WiFi/CDMA450 network with cellular 
handsets as end user devices is economically viable even with nearly full subsidization of handsets. Limited affordability of handsets has traditionally hindered adoption of communications services in rural developing regions. Finally, costs linked to regulatory policies constitute a substantial portion of the overall network cost structure. Any reduction in such costs (e.g. lower termination rates) has a significant beneficial impact on network economics.

\section{Defining the rural setting}

The rural connectivity landscape in developing countries presents a contrast to urban telecommunications in the developed world. Three aspects are noteworthy. First, any networking technology needs to be affordable. Lowering cost at the expense of reliability (e.g., no backup equipment, intermittent connectivity) and sharing of end-user devices ( e.g., Grameen phone [11], kiosks) may be an acceptable trade-off. Second, coverage is more important than capacity. Urban settings are capacity limited. Service providers must place multiple base stations in a small area to cater to large volume of traffic. In contrast, rural settings are coverage limited. Service providers would like a single base station to cover as large a geographical area (i.e., as many users) as possible. Finally, demand is difficult to forecast. There is no real measure of demand in rural areas. However a few trends point towards a latent demand and a willingness to invest in telecommunications. For example, rural spending in China has risen threefold from 1990 to 2002 while the percentage spent on telecommunications and transportation (on an absolute level) has risen from $1 \%$ of overall spending to $6 \%$ of overall spending. A similar trend is visible in Bangladesh where rural residents devote $7 \%$ of their income to telecommunications.

Telecommunication markets can be classified according to bandwidth demand per user (peak and average demand), purchasing power per user and the population density. Areas with a high population density and high bandwidth demand per user can be serviced by fiber. On the other hands areas with low population density and high purchasing power per user (e.g., Alaska, USA) can be serviced by satellite.

The rural environment in developing countries is characterized by medium to low population densities, low bandwidth demand per user and very low purchasing power per user. Hence any technology to provide connectivity to these regions must be characterized by low cost per unit of demand.

\subsection{Economic model for analysis}

Expenditure associated with any telecommunications deployment can be classified into two categories: Capital Expenditure (CapEx) and Operating Expenditure $(O p E x)$. CapEx covers the basic infrastructure for providing connectivity. In the case of a wireless network like CDMA450, CapEx will include the cost for towers, network equipment, primary or backup power sources, installation costs, one-time spectrum licensing fees etc. CapEx can either be viewed as a one-time investment or as a recurring investment where the infrastructure continues to grow over time. OpEx, on the other hand, is the cost expended by the service 
provider for operating and maintaining the network and supporting users. For example, OpEx includes the salaries of employees, recurring power costs, recurring spectrum licensing fees and per call termination charges.

Note that our analysis applies to the entire ecosystem for providing connectivity to rural environments irrespective of the specific allocation of economic interests between various parties in the ecosystem. Our interest is in analyzing the overall economic viability of this market and in highlighting the key technological and economic conclusions that effect the value chain in the aggregate. The impact of external subsidies may also be explored within this framework.

Economic terms: Throughout this paper, we use two standard economic measures, namely the Net Present Value (NPV) and Internal Rate of Return $(I R R)$, for evaluating the economic viability of different technologies. Over a specific time-period $T$ and an appropriate discount rate $r, \mathrm{NPV}$ is the sum total of the future stream of revenues and expenses discounted to the present. If the NPV over time-period $T$ is negative, the investment is deemed not profitable. Over a time-period $T$, IRR represents the value of the discount rate $r$ at which NPV becomes zero. Detailed definitions of economic terms used in this paper can be found in [12].

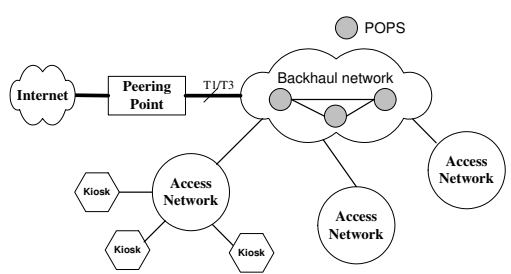

(a)

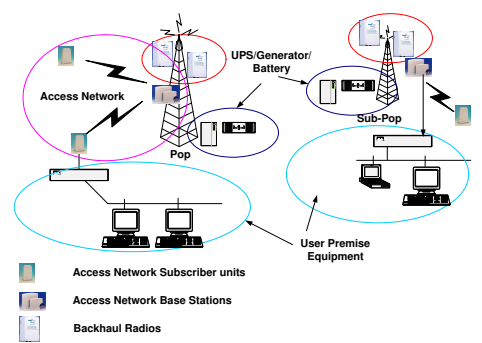

(b)

Fig. 1. (a)Basic network architecture for providing connectivity comprises a Backhaul network for connecting to the worldwide network and a set of Access networks for connecting to individual users/kiosks. (b) Corresponding elements in the Akshaya network

\section{$3 \quad$ Networking technologies for the rural setting}

To provide connectivity to a rural community, a peering/transit/exchange point provided by a standard telecommunications provider must be linked to users either using kiosks or cybercafes. This linking process is provided by two different classes of technologies: Access technologies that are used to connect individual users/centers to Points of Presence (POPs) of the network and Backhaul technologies that are used to link POPs to the worldwide network. The basic structure of such a network shown in Figure 1(a).

For our analysis we consider four access technologies: WiMax, CDMA450 (CDMA2000 at $450 \mathrm{MHz}$ ), Enhanced WiFi (WiFi with high gain unidirectional antennas or directional antennas), and WipLL (see Section 4 for details). A further description of these technologies can be found in [12]. Here we reproduce relevant characteristics and costs of these four technologies (Table 1). We make 


\begin{tabular}{|l|l|l|l|l|}
\hline & WiMax & CDMA450 & WiFi & WipLL \\
\hline Coverage & $5 \mathrm{~km}$ & $17 \mathrm{~km}[7]$ & $\begin{array}{l}1-2 \mathrm{~km} \text { (omni) } \\
5 \mathrm{~km} \text { (directional) }\end{array}$ & $5 \mathrm{~km}$ \\
\hline $\begin{array}{l}\text { Number of } \\
\text { simultaneous users }\end{array}$ & 16 & $\begin{array}{l}32 \text { on each } \\
1.25 \mathrm{MHz} \text { carrier }\end{array}$ & $\begin{array}{l}3 \text { for } 802.11 \mathrm{~b} \text { (directional) } \\
24 \text { for } 802.11 \mathrm{a} \text { (directional) }\end{array}$ & 126 total \\
\hline $\begin{array}{l}\text { Spectrum } \\
\text { Costs }\end{array}$ & $\begin{array}{l}\text { unlicensed }(2.4 / 5 \mathrm{GHz}) \\
\text { licensed }(3.5 \mathrm{GHz})\end{array}$ & licensed & unlicensed & unlicensed \\
\hline $\begin{array}{l}\text { Spectrum } \\
\text { availability }\end{array}$ & $\begin{array}{l}380 \mathrm{MHz} \text { at } 2.4 \mathrm{GHz}[13 \\
200 \mathrm{MHz} \text { at } 3.5 \mathrm{GHz}[13] \\
150 \mathrm{MHz} \text { at } 5 \mathrm{GHz}[13]\end{array}$ & $9 \mathrm{MHz}$ FDD & $\begin{array}{l}80 \mathrm{MHz} \text { at 2.4GHz }[13] \\
580 \mathrm{MHz} \text { at } 5 \mathrm{GHz}[13]\end{array}$ & Same as WiFi \\
\hline $\begin{array}{l}\text { Peak throughput } \\
\text { per user }\end{array}$ & $4 \mathrm{Mbps}$ & $1 \mathrm{Mbps}[7]$ & $\begin{array}{l}802.11 \mathrm{~b}: 5-7 \mathrm{Mbps} \\
802.11 \mathrm{a}: 20-30 \mathrm{Mbps}\end{array}$ & $4 \mathrm{Mbps}$ \\
\hline $\begin{array}{l}\text { Base station costs } \\
\text { current, projected }(2 \mathrm{yrs})\end{array}$ & $\begin{array}{l}\$ 1,999, \\
\$ 1,000\end{array}$ & $\begin{array}{l}\$ 20,000, \\
\$ 10,000\end{array}$ & $\begin{array}{l}\$ 500, \\
\$ 300\end{array}$ & $\begin{array}{l}\$ 2,100, \\
\$ 1,500\end{array}$ \\
\hline $\begin{array}{l}\text { Subscriber unit costs } \\
\text { current, projected }(2 \mathrm{yrs})\end{array}$ & $\begin{array}{l}\$ 1,199 \\
\$ 600\end{array}$ & $\begin{array}{l}\$ 250, \\
\$ 150\end{array}$ & $\begin{array}{l}\$ 500, \\
\$ 300\end{array}$ \\
\hline
\end{tabular}

Table 1. A comparison of capabilities and costs of various Access technologies

two assumptions about access technologies in our analysis: Firstly, we expect the cost for subscriber units for standardized technologies (WiMax, WiFi) to be similar once deployment is widespread (see [12] for operating case assumptions). Secondly, technologies operating in the same spectrum will have similar range. Differences in average bandwidth per user may arise from differences in the Medium Access Control (MAC) schemes employed by each technology.

For backhaul Technologies we consider the three technology options: Extended range WiFi (WiFi with directional antennas), Fiber (Passive Optical Networks) and VIP (proprietary technology from Wi-LAN used in the current Akshaya network). These technologies are further explained in [12].

\section{Akshaya - a case study}

The Akshaya project is a large rural wireless network [14] developed as a joint project between Tulip IT [15] and the Government of Kerala, India, to provide connectivity to the Malappuram district. The aim of this project is to provide connectivity to a group of people rather than individual users. 630 Akshaya centers (one for every 2000 families) are located throughout the district. The estimated costs for deployment are roughly a dollar per covered population. Each Akshaya center (AC) is setup and maintained by local entrepreneurs who receive a subsidized loan from the Government. These sites also provide computer training to one member of each household. Each entrepreneur pays Tulip a flat fee of $\$ 20$ per month for network connectivity.

The Akshaya network consists of wireless backhaul links based on patented VINE (Versatile Intelligent Network Environment) technology from Wi-LAN, Canada [10]. This Technology has been incorporated in the VIP radios from Wi-LAN. Each VIP radio can serve as a basestation (using sectored antennas) or as a repeater (using directional antennas). A single radio connects to the uplink node in the backhaul network. A second radio communicates with all the downlink nodes. These two nodes are bridged using a Wireless Ethernet Bridge. Each radio has $1 \mathrm{~W}$ transmission power and provides $11 \mathrm{Mbps}$ in the $2.4 \mathrm{GHz}$ band. 
For connecting the backhaul network with the Akshaya Centers, (point to multi-point links) Tulip used WipLL technology from Marconi and Airspan [8]. Each WipLL basestation serves up to 127 centers using sectored or omni antenna.

An analysis of the Akshaya network was used to determine the key network elements required to install such a network. These network elements are depicted in Figure 1(b) and explained in Table 2. Table 2 also lists the components located as each network node.

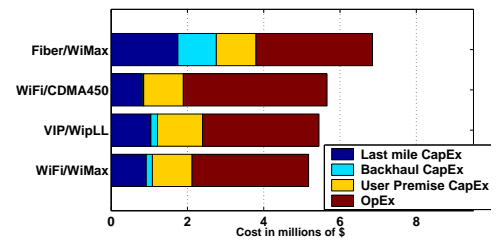

(a)

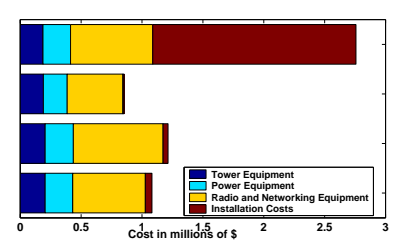

(b)

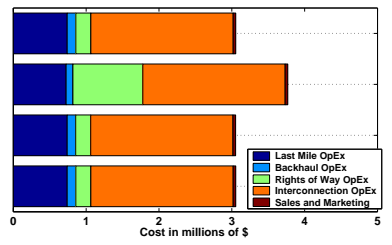

(c)

Fig. 2. Total costs, CapEx and OpEx for building the Akshaya network using various technology options.

\subsection{Economic Analysis of the Akshaya network}

The network CapEx and OpEx was split into three main categories: Backhaul, Last Mile and User premise.

Backhaul: The backhaul costs consists of all backhaul radio equipment, POP tower costs, auxiliary power costs and associated installation and maintenance costs. Land lease costs are factored in as Operating expenses.

Last mile: The Last Mile costs consists of all access (base station and subscriber side equipment) radio equipment, subPOP tower costs, auxiliary power costs and associated installation and maintenance costs.

User Premise: The User Premise Equipment (UPE) costs consist of $A k$ shaya center networking equipment and computer costs.

\begin{tabular}{|l|l|l|}
\hline Key terms & Explanation & Equipment \\
\hline POP & $\begin{array}{l}\text { A POP hosts backhaul links and } \\
\text { an Access Base Station for } \\
\text { connecting to Akshaya centers. }\end{array}$ & $\begin{array}{l}n \text { Backhaul radios, 1 30m Tower } \\
\text { 1 Access Base Station, Shelter } \\
\text { UPS with battery, Generator/Solar }\end{array}$ \\
\hline subPOP & $\begin{array}{l}\text { Akshaya centers which } \\
\text { also serve as POPs }\end{array}$ & $\begin{array}{l}n \text { Backhaul radios, 1 9m Tower } \\
\text { 1 Access Base Station, Shelter } \\
\text { UPS with battery, Generator/Solar }\end{array}$ \\
\hline relayPOP & $\begin{array}{l}\text { Backhaul relay towers that do not } \\
\text { host Access basestations - } \\
\text { used to connect POPs }\end{array}$ & $\begin{array}{l}n \text { Backhaul radios, 1 30m Tower } \\
\text { Generator } / \text { Solar }\end{array}$ \\
\hline AC & Village kiosk with 3 to 5 PCs & $\begin{array}{l}\text { 1 Access Subscriber unit, Ethernet switch } \\
\text { 3-5 PCs, UPS with battery }\end{array}$ \\
\hline
\end{tabular}

Table 2. List of equipment at each node.

We calculated the total CapEx and OpEx using the following Backhaul/Last mile technology pairs: WiFi/WiMax, WIP/WipLL, Fiber/WiMax and WiFi/CDMA450. 


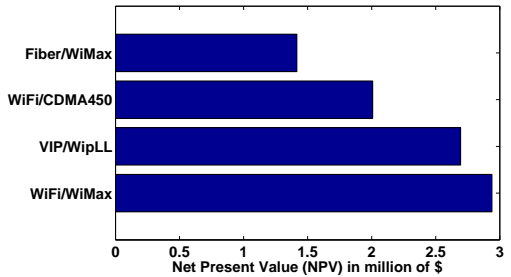

(a)

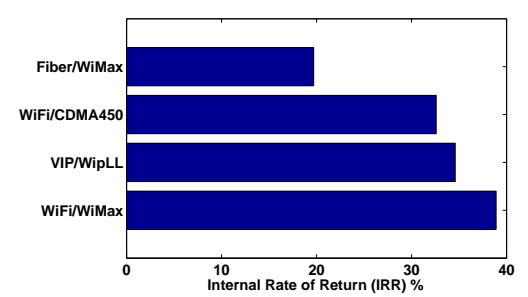

(b)

Fig. 3. (a)Net Present Value (NPV) for various technology options (b) Internal Rate of Return (IRR) for various technology options

All scenarios except WiFi/CDMA450 use 18 POPs and 16 subPOPS. CDMA450 requires 3 POPS and 3 subPOPS since a lower frequency provides a larger coverage area. Figure 2(a) shows the CapEx and OpEx (for 5 years) for each of the four technology options. The CapEx has been split into Last Mile CapEx, Backhaul CapEx and User Premise CapEx. Alternatively CapEx can be separated into tower cost, primary and/or auxiliary power equipment cost, radio and networking equipment cost and installation cost which can be seen in Figure 2 (b).

Similarly, the OpEx can be classified under different categories: Last Mile OpEx (Power cost and maintenance of subPOPs), Backhaul OpEx (Power cost and maintenance of POPs), Rights of Way OpEx (Tower leasing cost, Spectrum leasing cost and miscellaneous government fees), Interconnection OpEx (Termination and leased line cost) and Sales and Marketing cost. This OpEx breakdown for different technology options can be seen in Figure 2(c). The key assumptions made in generating these figures can be found in [12]. The final NPV and IRR for all technology options can be found in Figure 3. The Time for Cash-flow Profitability was 1 year for all technologies except WiFi/CMDA450 which required 2 years due to a larger OpEx. Time to Break even was 4 years for all technologies.

Key items to note in the cost breakdown include:

1. For the backhaul, wireless (both WiFi and VIP) technologies have at most one-eight the backhaul CapEx as compared to the fiber scenario. While WiFi radio costs are lower than VIP, radio costs in general comprise a small portion of the overall backhaul costs and hence this difference is not significant.

2. The CDMA450 scenario exhibits the lowest CapEx since the number of POPs and subPOPS is greatly reduced. However spectrum costs ${ }^{1}$ weigh heavily in the OpEx reducing the final NPV. This can be seen in the Rights of Way OpEx for CDMA450 in Figure 2(c).

3. The Fiber scenario is based on Passive Optical Network (PON) technology using a single chassis at the peering point with two line-cards. The fiber is assumed to be strung along towers due to the hilly terrain. The bulk of the costs (three-fourths of a million dollars) is to string the fiber on towers.

\footnotetext{
${ }^{1}$ In India spectrum licensing costs are a percentage of service revenues. In our analysis we assume the licensing costs for the $450 \mathrm{MHz}$ band would be similar to traditional cellular spectrum bands.
} 
4. Termination costs $(\$ .005 /$ minute) account for a large portion of the OpEx since we assume that a majority of the traffic is voice calls which terminate on regular telephone networks.

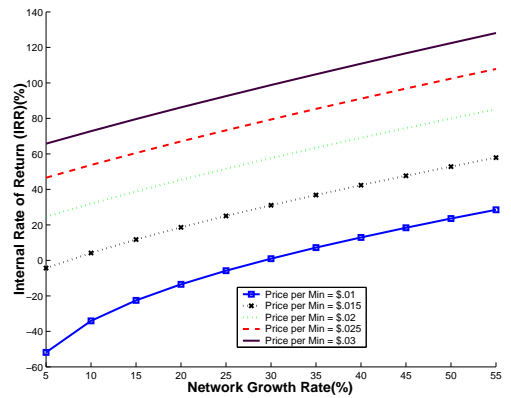

(a)

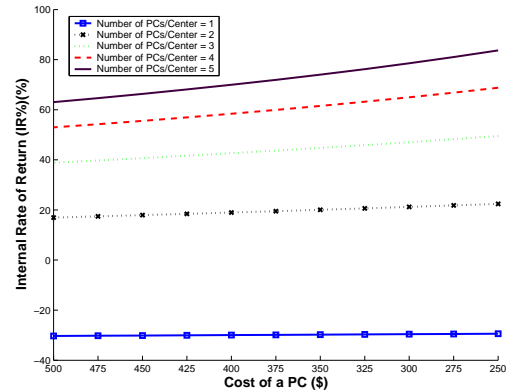

(b)

Fig. 4. (a)Effect of network utilization growth rate and price per minute on the IRR. For all network utilization growth rates, a minimum price of $\$ 0.02$ per minute is required for profitability. (b)Effect of changing cost per PC and the number of PCs per Akshaya center on the IRR. A minimum of 2 PCs per center are required for profitability.

\subsection{Sensitivity Analysis for Akshaya}

While our model is based on data from Tulip IT, and other publicly available information, this information is a rough estimate since prices in this industry change frequently. That being the case, we decided to perform sensitivity analysis to determine the impact of changes in our assumptions on the projected financial returns from the project. This is of particular importance for the revenue drivers, given the uncertainty of demand in many rural areas. Hence we considered the effect of the price per minute and the network utilization growth rate on the net IRR for the technology choice of WiFi with WiMax. This analysis is shown in Figure 4(a). For all network utilization growth rates, the minimum price of $\$ 0.02 /$ minute is required for profitability. Since the termination cost is $\$ .005 /$ minute, net value to the service provider is $\$ 0.015 /$ minute in this case.

Similarly since User Premise Equipment comprises the majority of the CapEx in all cases except the fiber scenario, we studied the variation of the number of PCs per Akshaya center and the cost per PC on the IRR. It is worth noting that we assume network usage to be proportional to the number of PCs per Akshaya center. Again, a minimum of 2 PCs per center are required for a positive value of IRR (See Figure 4(b)). In other words, each Akshaya center needs sufficient demand to support at least 2 PCs to achieve a positive IRR (even with no termination cost and a free PC, a single PC would not yield positive IRR).

\section{Discussion}

Alternative to Sharing: Use of individual devices: In Section 2, we used the kiosk/cybercafe model to leverage the aggregate purchasing power of rural 
communities. While sharing of devices reduces the cost per user, it also limits the demand per user. In order to evaluate the feasibility of distributing individual devices to users, we focused on the CDMA450 case where the 6 Base stations atop the POPs and subPOPs were converted into regular cellular base stations for voice calls. Each CDMA basestation with 6 sectored antennas can serve 1000 customers. Under this model the 630 Akshaya centers are assumed to be cellular handset retailers. A usage of 2 min per day per subscriber was assumed. This was increased by 1 min each year to 6 minutes per day per subscriber in year 5 . We neglected any upfront spectrum licensing costs for the $450 \mathrm{MHz}$ band. The subscribers were charged $\$ 0.02$ per minute and received a subsidy of $\$ 30$ on each handset.

In this scenario, recurring spectrum licensing cost represents $26 \%$ of the OpEx and $11 \%$ of the revenue. The IRR in this case is $28.5 \%$ which rises to $44.8 \%$ if recurring spectrum licensing cost is neglected. Assuming 100, 000 handsets are in use (a penetration level of $1.58 \%$ ), the highest handset subsidy possible to achieve break even is $\$ 38$ when recurring spectrum licensing costs are taken into account. This subsidy rises to $\$ 45$ if recurring spectrum licensing costs are omitted. In fact, neglecting recurring spectrum costs gives the handset scenario a better IRR than the kiosk model. With handset prices entering the sub $\$ 50$ price range, this analysis suggests that handsets could be nearly fully subsidized at these usage levels and penetration rates while still achieving economic viability for the network.

Extending the Akshaya analysis: In most respects the Akshaya case represents a worst case scenario. Malappuram is a hilly region with extremely dense vegetation. Hence, Tulip has to use high towers to avoid this vegetation and get a line of sight to a POP or subPOP. If we assume a similar population density but a flat terrain, tower heights can be reduced. However, this reduction does not impact the analysis in any significant manner. If tower heights were to be reduced by half for the $\mathrm{WiFi} / \mathrm{WiMax}$ scenario, the IRR only increases by $1.8 \%$. Change in terrain/frequency which enables a reduction in number of towers has a more tangible impact on the CapEx. If we could double the coverage area and hence halve the number of POPs and subPOPs, the IRR would increase to $2.7 \%$.

Impact of Regulation: In developing countries, telecommunication markets are usually highly regulated across several dimensions including spectrum allocation, approval for rights of way and service areas, universal service provisions, termination rules and rates, business licenses, service import tariffs. Regulation can play a crucial factor in determining the economic viability of different technologies since it effects a variety of critical business processes. For example, for the WiFi/WiMax scenario, regulatory costs in total represent $45.5 \%$ of the overall CapEx and 5 year OpEx. Eliminating regulatory costs would increase the IRR by $74.5 \%$.

As illustrated in Figure 2(c), termination rates make up a significant portion of the OpEx. Reducing the termination rate allows service providers to lower their price which in turn is likely to increase usage due to elasticity of demand. We studied the effect of a reduction of termination charges by $\$ .001$ every year. We varied the demand elasticity from 0 to -1 (i.e. a price elasticity of $-x$ implies that a price reduction of $x \%$ would increase network utilization by $x \%$ over and 
above the assumed ramp in network utilization). This analysis reveals that the IRR increases from $40.8 \%$ to $55.2 \%$ as the elasticity is increased from 0 to -1 .

\section{Conclusions}

The economic viability of communications networks in rural developing regions with severe affordability constraints, low population densities, and uncertain demand has long been a concern. This paper presents the case for economically viable networks in rural developing regions. For the Akshaya case study, we show that a wireless network using WiFi for the backhaul, CDMA450 for the access network, and shared PCs for end user devices has the lowest deployment cost. Once the expected spectrum licensing cost for CDMA450 is included, a network with lease exempt spectrum using WiFi for the backhaul and WiMax for access is the most economically attractive option. However, even with the WiFi/WiMax scenario, regulatory costs comprise nearly half the total cost structure of the network demonstrating the significant impact of regulatory policies on network economics. Finally, we demonstrate the business case for a WiFi/CDMA450 network with nearly fully subsidized cellular handsets as end user devices. Such a network overcomes the handset affordability constraint which has traditionally hindered adoption of communications services in rural developing regions.

\section{Acknowledgment}

This material is supported by the National Science Foundation under Grant No. 0326582. The authors gratefully acknowledge the support of Tom Kalil, Drew Isaacs, Bob Cole, the Management of Technology Program and the Technologies and Infrastructure for Emerging Regions (TIER) group at UC Berkeley.

\section{References}

1. http://www.who.int/pbd/blindness/onchocerciacis/en.

2. O. Badiane and G.E. Shively. Spatial Integration, Transport costs, and the Response of Local Prices to Policy Changes in Ghana. Journal of Development Economics, 56:411-431, 1998.

3. http://www.mp.nic.in/gramsampark/.

4. C.K. Prahalad. Fortune at the bottom of the pyramid: Eradicating poverty through profit. Technical report, Wharton School Publishing, 2005.

5. http://www.wi-fi.org.

6. http://www.wimaxforum.org/home.

7. Asian Telco Technology Primer - CDMA450: Perpetuating Asia's Growth + Profitability Story? Technical report, Lehman Brothers, June 10.2004.

8. http://www.airspan.com/products/wipllsolution.htm.

9. http://www.ponforum.org.

10. http://www.wi-lan.com.

11. http://www.grameenphone.com.

12. Mishra S. M. et al. Economic Analysis of Networking Technologies for Rural Developing Regions. Technical Report UCB/CSD-05-1411, University of California Berkeley, September 2005.

13. G. Wu. Using the RF Spectrum for WiMAX. EEtimes, May 2005.

14. http://www.akshaya.net.

15. http://www.tulipit.com. 